\title{
As universidades públicas portuguesas \\ e a capacitação na área da Educação em Ciências no âmbito da cooperação internacional para o desenvolvimento: do mapeamento à problematização
}

\author{
Betina Silva-Lopes
}

\begin{abstract}
Resumo:
Neste artigo identifica-se e problematiza-se o contributo que as universidades públicas portuguesas (UPP) têm dado no domínio da capacitação, na área das Ciências, na qualidade de agentes de cooperação para o desenvolvimento, nomeadamente através de protocolos estabelecidos com os seis países de Língua Portuguesa da região de África, Caraíbas e Pacífico (ACP). Metodologicamente a investigação identifica-se como estudo misto sequencial. A primeira fase do estudo consistiu no levantamento de 189 protocolos (2000-2015) e respetiva categorização, a partir da análise da informação disponível nas páginas oficiais das UPP. Na segunda fase da investigação, assente na metodologia de estudo de caso, identificam-se e discutem-se as principais fragilidades e ameaças referidas por um conjunto de 17 profissionais universitários de uma UPP no âmbito de dois protocolos de cooperação (cluster). Os testemunhos foram recolhidos através de inquérito por entrevista individual semiestruturada. Os resultados apontam, por um lado, para um papel de relevo das UPP no domínio da Educação em Ciências, em particular na formação de professores. Por outro, é consolidada a perceção da importância de investir em estratégias de formação na área da cooperação internacional para o desenvolvimento (CID) dirigida a profissionais universitários e, ainda, em estratégias de disseminação do trabalho desenvolvido, no sentido de potenciar a qualidade e o impacto de protocolos futuros.
\end{abstract}

Palavras-chave:

cooperação portuguesa para o desenvolvimento; países de Língua Portuguesa; região de África, Caraíbas e Pacífico (ACP); capacitação em Ciências; universidades públicas. 


\title{
Mapping and problematizing the role of Portuguese public universities towards Science Education in the context of international cooperation for development
}

\begin{abstract}
This article identifies and questions the contribution that Portuguese public universities (PPU) have made in Sciences and Science Education, as agents of development cooperation, namely through protocols established with institutions from the six Portuguese-speaking countries in the Africa, Caribbean and Pacific (ACP) region. Methodologically the investigation is identified as a mixed sequential study. The first phase of the study consisted in a survey of 189 protocols (2000-2015) and respective categorization, based on the analysis of the information available on the official webpages of the PPU. In the second phase of the investigation, based on a case study methodology, the main weaknesses and threats mentioned by a group of 17 university professionals from one PPU are identified and discussed. The testimonies, associated to two cooperation protocols (cluster), were collected through semi-structured individual interviews. Results point to a prominent role of PPU in the field of Science Education, particularly in teacher training considering the six Portuguese speaking countries of the ACP region. Furthermore, the importance of investing in academic staff training strategies, in the area of international cooperation for development (CID), emerged. Finally, in order to enhance quality and impact of future protocols it is also important to invest in broader dissemination strategies of the work that has been developed so far.
\end{abstract}

Keywords: Portuguese cooperation for development; Portuguese-speaking countries; Africa, Caribbean and Pacific (ACP) region; Sciences and Science Education; public universities.

\section{Les universités publiques portugaises et la formation dans le domaine de l'enseignement des sciences dans le cadre de la coopération internationale pour le développement: de la cartographie à la problématisation}

Résumé: Cet article identifie et questionne la contribution des universités publiques portugaises (UPP) au niveau de la formation, dans le domaine des sciences, en tant qu'agents de la coopération au développement, notamment au travers des protocoles établis avec les six pays lusophones de la région Afrique, Caraïbes et Pacifique (ACP). Du point de vue méthodologique, l'enquête est identifiée comme une étude séquentielle mixte. La première phase de l'étude s'appuie sur la collecte de données provenant des 189 protocoles (2000-2015) et leur catégorisation respective, sur la base de l'analyse des informations disponibles sur les pages officielles des UPP. Dans la deuxième phase de l'enquête, sur la base de la méthodologie des études de cas, les principales faiblesses et menaces mentionnées par un groupe de 17 professionnels universitaires d'une UPP sont identifiées et discutées dans le cadre de deux protocoles de coopération (cluster). Les témoignages ont été recueillis lors d'un entretien individuel semi-structuré. Les résultats indiquent, d'une part, un rôle de premier plan de I'UPP dans le domaine de l'enseignement des sciences, en particulier dans la formation des enseignants. D'autre part, la perception de l'importance d'investir dans des stratégies de formation dans le domaine de la coopération internationale pour le développement (CID) à destination des professionnels universitaires est consolidée, ainsi que des stratégies de diffusion des travaux développés, afin d'améliorer la qualité et l'impact de futurs protocoles.

Mots-clés: coopération portugaise pour le développement; pays lusophones; région Afrique, Caraïbes et Pacifique (ACP); formation en Sciences; universités publiques.

\section{Universidades públicas portuguesas y formación en Educación en Ciencias en el ámbito de la cooperación internacional para el desarrollo: del mapeo a la problematización}

Resumen: Este artículo identifica y cuestiona la contribución que las universidades públicas portuguesas (UPP) han hecho en el campo de la capacitación, en el área de las Ciencias, como agentes de cooperación para el desarrollo, a través de protocolos establecidos con seis países de habla portuguesa en la región de África, Caribe y Pacífico (ACP). Metodológicamente, la investigación se identifica como un estudio secuencial mixto. La primera fase de estudio consistió en la recolección de 189 protocolos (2000-2015) y su respectiva categorización, basada en el análisis de la información disponible en las páginas oficiales de las UPP. En la segunda fase de la investigación, basada en la metodología del estudio de caso, las principales debilidades y amenazas mencionadas por un grupo de 17 profesionales universitarios de una UPP se identifican y discuten en el ámbito de dos protocolos de cooperación (cluster). Los testimonios fueron recopilados a través de una encuesta por entrevista individual semiestructurada. Los resultados apuntan, por un lado, a un papel destacado de la UPP en el campo de la Educación en Ciencias, particularmente en la formación de docentes. Por otro lado, se consolida la percepción de la importancia de invertir en estrategias de capacitación en el área de la cooperación internacional para el desarrollo (CID) dirigida a profesionales universitarios, así como estrategias para la difusión del trabajo desarrollado, con el fin de mejorar la calidad bien como el impacto de futuros protocolos.

Palabras clave: cooperación portuguesa para el desarrollo; países de habla portuguesa; región África, Caribe y Pacífico (ACP); formación en ciencias; universidades públicas. 


\section{Introdução}

Nos últimos anos as instituições de ensino superior (IES) têm-se envolvido num processo de internacionalização sem precedentes (Brandenburg \& de Wit, 2015; GuiIherme \& Dietz, 2015). Em estreita articulação com o "movimento da internacionalização" das IES, simultaneamente potenciado pela globalização e potenciador da globalização, os protocolos de cooperação entre IES de países desenvolvidos e instituições e organizações de países em desenvolvimento têm crescido substancialmente (Alonso \& Glenni, 2015; Hamza, 2010; Lopes \& Diogo, 2019).

Também as IES portuguesas se têm assumido cada vez mais como agentes de desenvolvimento, potenciado pelo modelo de cooperação descentralizado que Portugal tem vindo a adotar (Faria, 2014; Sangreman, 2015). Apesar do número crescente de protocolos, continua a faltar um diálogo sistemático e construtivo dos esforços de cooperação que têm sido levados a cabo pelos diversos agentes de cooperação (Ferreira, Faria \& Cardoso, 2015), entre os quais as IES portuguesas, de forma individual e coletiva (Alves, Marques \& Saur, 2004; Darling-Hammond, 2017).

Reconhecendo que as sociedades atuais são cada vez mais moldadas pela Ciência e pela Tecnologia, e de que um desenvolvimento equitativo e inclusivo implica uma educação científica de qualidade, foi desenvolvido entre 2015 e 2018 um projeto investigativo focado no estudo do trabalho desenvolvido pelas Universidades Públicas Portuguesas (UPP) no domínio da Educação em Ciências ${ }^{1}$, em particular na formação de professores de Ciências. De facto, a Educação em Ciências desempenha um papel determinante na formação de cidadãos críticos e ativos (Hodson, 2003), constituindo-se, por isso, como um bem público e logo um tema de relevância crucial ao nível das políticas educativas nacionais, regionais e internacionais (Martins, Pedrosa, Ferreira \& Simões, 2014).

O estudo, cujos resultados se discutem parcialmente neste artigo, foi desenvolvido a partir dos seguintes objetivos investigativos:

- (i) delimitar e caracterizar a cooperação portuguesa, sob responsabilidade das UPP na área da formação e Educação em Ciências, desenvolvida com instituições congéneres dos seis países de língua oficial portuguesa da região de África, Caraíbas e Pacífico (ACP), nomeadamente Angola (ANG), Cabo Verde (CV), Guiné-Bissau (GB), Moçambique (MZ), São Tomé e Príncipe (STP) e Timor-Leste (TL);

- (ii) identificar e sistematizar conhecimento tácito adquirido por atores chaves no decorrer da implementação de protocolos de cooperação, no domínio da capacitação em Ciências, estabelecidos por uma UPP em particular (o "caso em estudo");

- (iii) delinear recomendações específicas para decisões e ações futuras no âmbito de protocolos de cooperação no domínio da Educação em Ciências, em particular, na formação de professores. 
O presente artigo encontra-se estruturado em quatro secções. Para além desta introdução, na qual se delimita o tema em estudo e se definem os objetivos investigativos, o artigo integra a secção da metodologia, na qual se explicita o desenho global do estudo, identificando-se os respetivos métodos de recolha e análise de dados. $\mathrm{Na}$ terceira secção, resultados, procede-se, num primeiro momento, à descrição do cenário global do trabalho realizado pelas UPP com países de língua oficial portuguesa da região de ACP. Num segundo momento, e a partir do cruzamento de testemunhos de atores envolvidos na capacitação de professores de Ciências, no âmbito de dois protocolos de uma UPP, discutem-se as principais fragilidades e ameaças associadas à concretização das tarefas e dos objetivos de trabalho relacionados com os protocolos de cooperação. Por fim, nas considerações finais, apresentam-se um conjunto de recomendações destinadas principalmente aos decisores institucionais no domínio em estudo.

\section{Metodologia}

A investigação foi desenvolvida entre 2015 e 2018 e assume-se, metodologicamente, como um estudo misto sequencial (Creswell, 2014), tendo sido desenvolvido em duas fases. Numa primeira fase, e no alinhamento do primeiro objetivo investigativo, procedeu-se ao levantamento dos protocolos estabelecidos, desde 2000, entre UPP e instituições congéneres dos países da ACP, no sentido de mapear o contributo das quinze UPP na arquitetura global do desenvolvimento 2 . O levantamento foi feito a partir da análise da informação e documentação disponibilizada nas páginas oficiais das UPP $^{3}$, em complemento com contactos pessoais, telefónicos e escritos (via e-mail), com informantes-chave (por exemplo, responsáveis pela coordenação de protocolos e diretores de departamento), sempre que considerado necessário. Foram identificados e categorizados um total de 189 protocolos $^{4}$ (Silva-Lopes, Albergaria-Almeida, Costa \& Callapez, 2016). De salientar que os protocolos considerados no estudo implicaram a deslocação de um ou vários docentes ao país parceiro. A UPP com o maior número de protocolos na área da formação de professores de Ciências $(n=6)^{5}$ foi selecionada para a segunda fase do estudo, tendo como base o pressuposto que nesta instituição haveria um maior número de atores com experiência de cooperação para o desenvolvimento, e, deste forma, com conhecimento tácito associado.

A segunda fase do estudo iniciou-se em janeiro de 2017, nomeadamente com a confirmação da possibilidade de participação dos principais intervenientes associados aos protocolos de cooperação da UPP selecionada. A recolha e análise sustentou-se na constituição de um cluster ${ }^{6}$ intrainstitucional de dois do total de protocolos associados à UPP (Figura 1). 


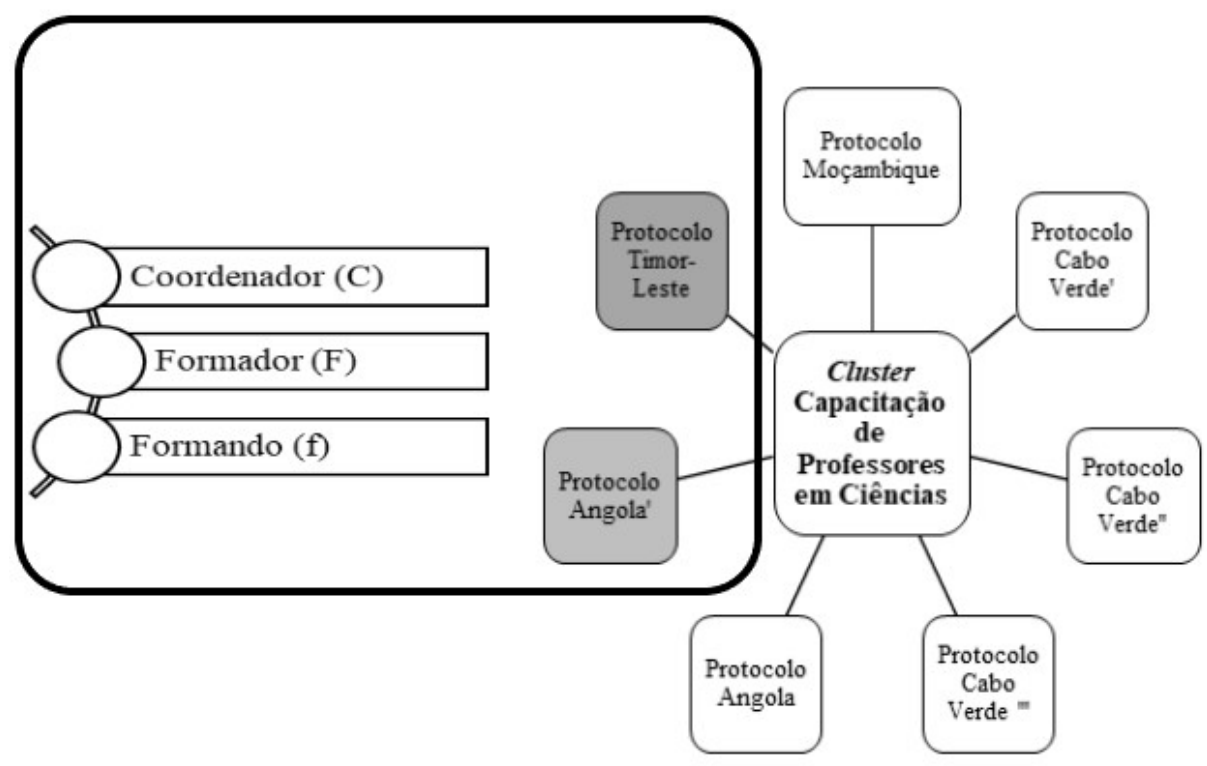

Figura 1. Enquadramento institucional do cluster em estudo

Ainda que tenham sido estudados apenas dois protocolos, é de referir que os mesmos tiveram um papel de relevo nos sistemas educativos dos países parceiros (Martins et al., 2014; Silva-Lopes et al., 2016). O critério de seleção dos dois protocolos, em detrimento dos restantes baseia-se no facto dos mesmos ainda estarem em vigor aquando a realização do presente estudo e da disponibilidade dos atores. A estratégia de articulação analítica, clustering, dos protocolos surge na linha da recomendação do Instituto Português de Apoio ao Desenvolvimento, IPAD (2010), da OCDE (2006; 2014) e de Sangreman (2015), na medida em que esta técnica de prospeção de dados potencia o cruzamento de experiências de aprendizagem e, desta forma, a inovação. No Quadro 1 encontra-se uma breve descrição dos dois protocolos que integram o cluster intrainstitucional, doravante designados de "Protocolo Angola" (PA) e "Protocolo Timor-Leste" (PTL). 
Quadro 1. Caracterização geral dos protocolos associados ao 'cluster' intrainstitucional

\begin{tabular}{|c|c|c|}
\hline & "Protocolo Angola" & "Protocolo Timor-Leste" \\
\hline Tipo e nível de ensino & $\begin{array}{l}\text { Formação Pós-graduada } \\
\text { (mestrado) de professores de } \\
\text { Ciências }\end{array}$ & $\begin{array}{l}\text { Formação inicial e contínua de } \\
\text { professores (de ensino pré secundário } \\
\text { e secundário) }\end{array}$ \\
\hline \multirow{2}{*}{$\begin{array}{l}\text { Área científica } \\
\text { Parceiro(s) institucional(ais) }\end{array}$} & $\begin{array}{c}\text { Ciências Básicas (Física, } \\
\text { Química, Matemática) e Ciências } \\
\text { da Vida (Biologia) }\end{array}$ & $\begin{array}{c}\text { Ciências Básicas (Física, Química, } \\
\text { Matemática) da Terra e da Vida } \\
\text { (Biologia e Geologia) }\end{array}$ \\
\hline & $\begin{array}{c}\text { Instituição de Ensino Superior } \\
\text { Pública }\end{array}$ & Ministério da Educação \\
\hline $\begin{array}{l}\text { Instituições } \\
\text { intermediárias/financiadoras }\end{array}$ & $\begin{array}{l}\text { Não se aplica } \\
\text { (protocolo direto) }\end{array}$ & $\begin{array}{c}\text { Fundação Calouste Gulbenkian } \\
\text { Camões - Instituto da Cooperação e } \\
\text { da Língua }\end{array}$ \\
\hline Início do protocolo & $\begin{array}{c}2009 \text { - em vigor à data do } \\
\text { estudo }\end{array}$ & 2012 - em vigor à data do estudo \\
\hline
\end{tabular}

A recolha de dados implicou inquérito por entrevista a 17 atores. A seleção dos entrevistados baseou-se no critério da diversificação de funções, designadamente funções de Coordenador (C), Formador (F) e formando (f). Foram entrevistados 4 coordenadores (2 do PTL e 2 do PA); 6 Formadores (3 do PTL e 3 do PA), e 7 formandos (6 do PA e 1 do PTL). No total das pessoas entrevistadas, salienta-se o seguinte:

- dois informantes, designadamente duas formadoras tinham experiência no âmbito dos dois protocolos em estudo;

- uma das formadoras tinha experiência de cooperação para o desenvolvimento, no âmbito de outros protocolos de capacitação em Ciências, mas estabelecidos por outra entidade que não a UPP selecionada. Os restantes informantes (Coordenadores e Formadores) não tinham experiência prévia de cooperação internacional para o desenvolvimento para além do âmbito dos dois protocolos em estudo.

Todas as entrevistas realizadas foram audiogravadas e integralmente transcritas, tendo sido posteriormente validadas pelos informantes. As transcrições validadas foram sujeitas a uma análise temática (Charmaz, 2014) com recurso a um software de análise qualitativa de dados (WebQDA®). A análise realizada partiu da técnica de análise SWOT, nomeadamente a identificação de aspetos positivos (eixo SO - Strengths \& Opportunities) e aspetos negativos (eixo WT - Weaknesses \& Threats) (Allouche \& Schmidt, 1999). 


\section{Resultados}

Nesta secção apresenta-se, primeiramente, um cenário global de capacitação no âmbito da Educação em Ciências em contexto de cooperação internacional, tendo como base 189 protocolos estabelecidos, nomeadamente entre UPP e instituições congéneres dos seis países de língua oficial portuguesa da região de ACP, no intervalo temporal de 2000-2015. De seguida, em articulação com os resultados da primeira fase do estudo, discutem-se as principais fragilidades e ameaças identificadas no contexto dos dois protocolos que constituem um cluster intrainstitucional de cooperação.

No que respeita ao mapeamento do contributo das UPP no domínio da cooperação para o desenvolvimento, dos 189 protocolos levantados, 80 foram estabelecidos no âmbito das Ciências Fundamentais (Ciências Básica e Ciência da Terra e da Vida, cf. nota 1), e 96 protocolos foram estabelecidos no domínio da Literatura e da Cultura Portuguesa ${ }^{7}$. De referir que 13 dos 189 protocolos não foram categorizados quanto ao domínio de cooperação, por serem acordos de natureza genérica ou pelo facto da informação acedida não ter sido suficiente para sustentar uma decisão de codificação. Do total dos protocolos levantados, 70 (37\%) tiveram apoio por parte do Camões I.P., entidade que tutela a cooperação portuguesa para o desenvolvimento ${ }^{8}$. Neste sentido, mais de metade dos protocolos levantados, nomeadamente $63 \%$ foram estabelecidos sem intervenção do Camões I.P., o que pode ser encarado como um reflexo do modelo descentralizado da cooperação portuguesa. De facto, este modelo tem permitido respostas nacionais e institucionais mais diversificadas, e desta forma, mais flexíveis (Sangreman, 2015), quando comparado com outros países em que os modelos são mais centralizados.

No âmbito da primeira fase do estudo, salienta-se ainda o aparente peso similar entre a capacitação no domínio da(s) Ciências (designadamente 42\%) e a divulgação da Literatura e da Cultura Portuguesa (51\%). Por um lado, estes dados podem ser encarados como indiciadores de um panorama divergente do descrito nos últimos dois pareceres do Comité de Ajuda ao Desenvolvimento da OCDE (2006, 2015), nos quais é enfatizada a "instrumentalização" da cooperação portuguesa para o desenvolvimento, nomeadamente, para a difusão da Cultura e da Língua Portuguesa. Por outro, importa refletir até que ponto os protocolos no domínio da Educação em Ciências contribuem (também) para a instrumentalização de uma cultura, na medida em que a capacitação é feita numa língua, que não é a nativa. Neste sentido, levanta-se a dúvida se uma das fragilidades da cooperação portuguesa será um enfoque "excessivo" na Cultura e Literatura Portuguesa ou se será, antes (ou também), um problema de visibilidade do trabalho que é desenvolvido no domínio da cooperação para além da difusão da cultura portuguesa, e para além da intervenção direta do Camões I.P., nomeadamente pelas UPP. De facto, durante o levantamento dos protocolos, verificou-se uma grande disparidade nas estratégias comunicacionais e, em alguns casos, lacunas na informação 
disponibilizada publicamente pelas UPP, o que limitou a constituição de uma base de dados exaustiva no domínio da formação em Ciências no contexto da cooperação.

Relativamente aos 80 protocolos de cooperação estabelecidos no domínio da Educação em Ciências (Figura 2), o maior número de protocolos identificado, foi estabelecido com Moçambique - MZ ( $n=27)$, seguido de Angola - ANG $(n=24)$ e depois por Cabo Verde - CV ( $n=18)$ - Figura 2a. No que respeita aos parceiros institucionais, mais de metade são IES públicas $(n=48)$, seguindo-se as instituições de ensino superior privadas $(n=22)$ - Figura $2 b$.

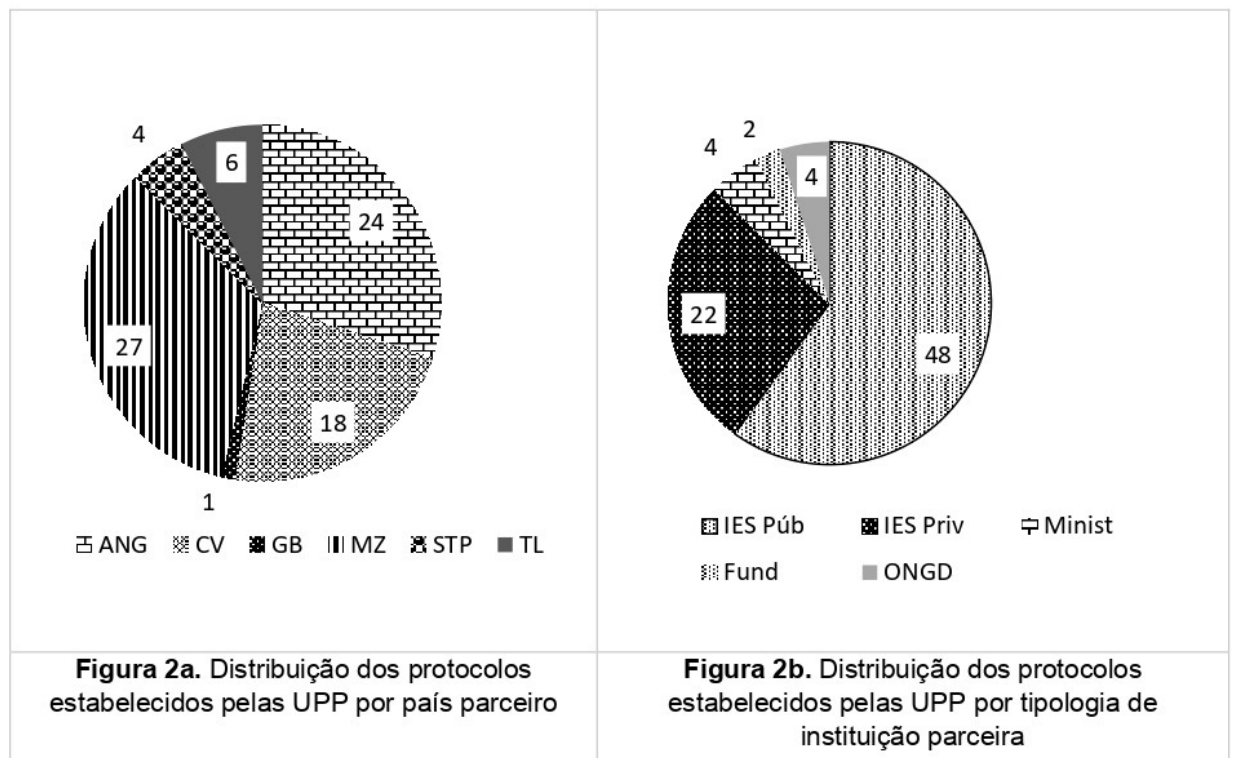

Figura 2. Cooperação para o desenvolvimento na área da Educação - um cenário global

Ainda no âmbito do mapeamento global do contributo das UPP na cooperação para o desenvolvimento, e com um enfoque particular na formação de professores de Ciências, foi possível identificar as quatro UPP com maior tradição/intervenção nesta área. Neste conjunto de UPP, que podem ser encaradas como tendo um papel de liderança na formação de professores de Ciências em contexto de cooperação, foi contactada a UPP com o maior número de protocolos estabelecidos neste domínio para integração na segunda fase do estudo.

Antes de se passar à discussão dos testemunhos dos informantes-chave entrevistados no âmbito do cluster intrainstitucional, e numa lógica de descrição do caso de estudo à luz do "mapa global" estabelecido na primeira fase investigativa, torna-se relevante referir que a delimitação do próprio cluster intrainstitucional reforçou a perceção de que 
o trabalho desenvolvido pelas UPP no domínio da Educação em Ciências se encontra sub-representado ao nível da base de dados, e estará muito provavelmente também "sub-representado" para a comunidade em geral. Isto é, da metodologia de trabalho seguida no âmbito deste estudo, emerge a perceção de que o contributo das UPP na cooperação para o desenvolvimento, e em particular no domínio da Educação em Ciências, é pouco visível, senão invisível, dentro e fora da academia. De facto, o contacto direto e (mais) prolongado com a instituição de origem do cluster intrainstitucional permitiu verificar que um dos dois protocolos em causa, designadamente o PA não tinha sido identificado no levantamento inicial, apesar do mesmo ter sido iniciado em 2009. A não identificação deste protocolo na base de dados deve-se ao facto de o mesmo não estar disponível no acervo público, isto é, na página oficial online, da UPP em causa. Estas lacunas de disseminação interna comprometem a disseminação intra e interinstitucional e, desta forma, o cruzamento de olhares e experiências de aprendizagem (conhecimento tácito) entre pares, uma das dificuldades mais referidas pelos entrevistados, e que tem sido igualmente enfatizada na literatura específica (Ferreira, Faria \& Cardoso, 2016; Lopes \& Diogo, 2019).

Os Quadros 2 e 3 sistematizam as ameaças e as fragilidades ${ }^{10}$ referidas pelos informantes. Limitações associadas à extensão do artigo determinaram a opção metodológica em focar a discussão apenas nas fragilidades e nas ameaças referidas pelos Coordenadores e pelos Formadores entrevistados em ambos os protocolos, numa lógica de que a sua referência nos dois contextos seja sinalizadora da sua natureza transversal, e desta forma, devam ser consideradas prioritárias em futuros diálogos intra e interinstitucionais.

No que respeita à categoria "ameaças" a falta de acesso a recursos no contexto de intervenção foi a dificuldade extrínseca mais referida, nomeadamente por 12 entrevistados. Ainda que não seja de estranhar esta dificuldade, uma vez que se está a falar de trabalho realizado com instituições parceiras localizadas em países em desenvolvimento, é de reforçar o facto de os $\mathrm{C}$ e dos $\mathrm{F}$ de ambos os protocolos sentirem esta dificuldade e em ambos os protocolos não ter sido dedicado um tempo prévio específico para o levantamento dos recursos e/ou de estratégias necessárias para a realização do trabalho associado ao protocolo, por exemplo através de um diálogo sustentado e especificamente focado na preparação das missões com os parceiros institucionais e, em última instância, com os beneficiários dos programas de formação. Esta dificuldade extrínseca (falta de recursos no contexto de formação/capacitação) estará intimamente associada à fragilidade mais referida pelos entrevistados, designadamente a "falta de preparação dos profissionais" nomeadamente para a coordenação de protocolos desta natureza e para a formação dos professores nestes contextos específicos. De facto, dos quatro coordenadores entrevistados, todos referiram que não tiveram preparação específica para esta área de trabalho, e que o convite para as funções se fundou na (sua) área de expertise científica. No entanto, e como tem vindo a ser enfatizado por estudos internacionais neste domínio, o trabalho no âmbito da cooperação internacional tem características e riscos muito próprios que importam ser trabalhados para 
evitar fazer "mais mal do que bem" (Goldstein, 2003; Gomes, 2018; Guilherme \& Dietz, 2015; Stein, Andreotti \& Susa, 2016). Em síntese e em articulação com o modelo definido em Silva-Lopes, Lucas, Albergaria-Almeida \& Martinho (2017), torna-se crucial "capacitar também os capacitadores", nomeadamente no sentido de os ajudar a lidar e a ultrapassar os demais constrangimentos associados à cooperação para o desenvolvimento, e dos quais as ameaças identificadas através dos testemunhos dos entrevistados, são ilustrativas (Quadro 4), designadamente a "falta de recursos humanos qualificados nas instituições parceiras" (A2, referida por nove entrevistados), a "ausência de memória institucional nas instituições parceiras" (A3, referida por três entrevistados), e a "não articulação do trabalho entre as diversas parcerias" (A4, referida por quatro entrevistados).

Quadro 2. Fatores externos negativos e excertos ilustrativos das entrevistas realizadas

\section{Ameaças}

A1 - falta de acesso a recursos no contexto de intervenção

$(n=12$, dos quais 8 de PTL e 4 de PA C2)

\section{Excertos ilustrativos}

"Foi muito frustrante ter de dar aulas sobre microscópia e ter apenas um microscópio funcional para trinta, quarenta formandos. Até havia mais alguns exemplares, mas nenhum deles funcionava. E é preciso pensar que o contexto de formação era um contexto privilegiado, na escola então é que não há mesmo nada." (PTL, F)

"E quando lá cheguei percebi que o contexto era ainda mais difícil. Porquê? Porque nos foi dito que iam ter acesso à net e não tinham net. Durante todo o tempo não tiveram. Cada um teve de se desenrascar..." (PA, F)

A2 - Falta de recursos humanos qualificados nas instituições parceiras

( $n=9$, dos quais 7 de PTL e 2 do PA)

A3 - Ausência de Memória Institucional (sistematizada) no respetivo parceiro

$(n=3$ dos quais 1 para PTL e 2 para PA)

A4 - Não articulação do trabalho entre as diversas colaborações que a Instituição parceira tem

$(n=2$, dos quais 1 para PTL e 1 para PA)
“ (...) na altura as instituições também perceberam que o IPAD e a Fundação Calouste Gulbenkian não têm massa crítica para fazer currículos" (PTL, C)

"O Ministério da Educação não tem capacidade para verificar se os memorandos caducaram (...) Tem que haver uma assessoria, mas, em última instância, quem assina por baixo (...) e é isto que eu acho que falha (...)" (PA, C)

"Um dos grandes desafios para nós é aceder a documentação, que eles não têm. A única, ou a pouca documentação." (PTL, C)

“Chegamos lá e nada estava organizado (...) havia uma sala ... e estava tudo no chão, aos montes e espalhado. Antes de mais passamos umas boas horas a organizar o que havia." (PA, C)

"Por exemplo, a meio da formação percebi que os professores usavam como apoio uns manuais cedidos por outros parceiros, cooperação brasileira, e tive que reformatar um pouco o que tinha planeado implementar, pois o trabalho prévio não estava articulado." (C2, F)

"No início da minha estadia achava que a coordenação de lá era pouco "sólida", mas ao longo das semanas contactei com outros cooperantes, isto é, com professores oriundos de outras instituições de outros países, e pensei...Pois de facto como gerir tudo isto? Esta instituição é um verdadeiro melting pot de escolas de pensamento e culturas de trabalho diferentes... é um vaivém constante de agentes de cooperação, assim é difícil manter uma linha de ação coesa..." 
Quadro 3. Fatores internos negativos e excertos ilustrativos das entrevistas realizadas

\begin{tabular}{|c|c|}
\hline Fragilidade & Excertos ilustrativos por dimensão \\
\hline $\begin{array}{l}\mathrm{F} 1 \text { - Falta de preparação } \\
\text { dos profissionais } \\
\text { (coordenadores/formadores/ } \\
\text { orientadores) para trabalhar } \\
\text { no contexto de cooperação } \\
\text { ( } \mathrm{n}=11 \text {, dos quais } 5 \text { para PTL } \\
\text { e } 6 \text { para PA) }\end{array}$ & $\begin{array}{l}\text { “(...) a tendência destes programas de cooperação é impormos sempre a } \\
\text { nossa perspetiva (...) Há um eurocentrismo muito grande, digam o que } \\
\text { disserem. Portanto, toda a colaboração da Europa nestes países há um } \\
\text { eurocentrismo marcante (...) e nós não somos sensibilizados para isso, } \\
\text { acabamos por aprender às nossas custas.” (PA, F) } \\
\text { "Porque uma coisa é ser expert no currículo, outra coisa é ser expert no } \\
\text { currículo em contexto de cooperação.” (PTL, F) } \\
\text { "Eu cheguei lá com as melhores das intenções... e mesmo assim, por } \\
\text { ingenuidade e ignorância acabei por falar da roda dos alimentos, quando } \\
\text { eles têm a sua própria pirâmide alimentar. Desconhecia isso, podia ter-me } \\
\text { preparado melhor" (PTL, F) }\end{array}$ \\
\hline
\end{tabular}

Fonte: autora

\section{Considerações finais}

A articulação entre os resultados das duas fases do estudo, a primeira de abordagem mais quantitativa e a segunda de abordagem mais qualitativa, permitiu a identificação de desafios-chave meritórios de uma reflexão alargada, nomeadamente ao nível das políticas de gestão institucional, a fim de potenciar sinergias entre os principais agentes de cooperação para o desenvolvimento no domínio da Educação em Ciências.

Face à meta 4.c. do objetivo 4 da Agenda 2030 da UNESCO, nomeadamente:

"By 2030, substantially increase the supply of qualified teachers, including through international cooperation for teacher training in developing countries, especially least developed countries and small island developing states" (UNESCO, 2016, p. 19),

prevê-se o aumento de protocolos estabelecidos no domínio em estudo a médio e longo prazo. Numa lógica de investigação aplicada, e de apoio à tomada de decisões futuras no domínio institucional, indo ao encontro do terceiro objetivo investigativo, são delineadas se seguintes recomendações/sugestões:

- as UPP, particularmente as que se assumem como líderes neste domínio, devem investir em estratégias de monitorização e avaliação dos protocolos de cooperação que estabelecem, no sentido de potenciar a recolha e partilha de aprendizagens,

- estratégias investigativas no domínio da cooperação para o desenvolvimento devem incluir a criação de recursos de apoio à gestão e tomada de decisão no âmbito dos protocolos de capacitação, assim como de estratégias de formação dos cooperantes e partilha sistemática de práticas e lições aprendidas no sentido de tornar tangível todo o conhecimento tácito que tem vindo a ser desenvolvido; 
- investir em estratégias de mapeamento e disseminação dos protocolos (mais) articuladas, no sentido de possibilitar diálogos interinstitucionais mais sustentados, e por outro, permitir a visibilidade, e logo valorização, deste trabalho pela comunidade em geral.

A operacionalização das recomendações anteriores poderá passar, por exemplo, pela criação de uma rede - cluster - no domínio da Educação em Ciência, e, particularmente, formação de professores, em contexto de cooperação internacional envolvendo as UPP e demais IES interessadas. A criação de um fórum específico, inteiramente focado na reflexão sobre o passado, presente e futuro da Educação em Ciências nos países da ACP poderá constituir-se como um fator determinante na formação de cidadãos críticos e ativos.

\section{Notas}

${ }^{1} \mathrm{O}$ conceito de Educação em Ciências (Science Education) adotado neste estudo parte da terminologia usada pela UNESCO (http://www.unesco.org/new/en/natural-sciences/special-themes/ science-education/) e inclui a educação em ciências básicas (Química, Física e Matemática), assim como a educação em Ciências da Terra e da Vida (Biologia e Geologia) e respetivas variantes de aplicação mais tecnológica (nomeadamente, as Engenharias).

${ }^{2}$ Em alusão ao estudo "O papel de Portugal na arquitectura global do desenvolvimento: opções para o futuro da Cooperação Portuguesa", feito por Ferreira, Faria e Cardoso (2015), e que corresponde ao primeiro esforço de sistematização do trabalho realizado pelos mais diversificados agentes da cooperação portuguesa. A recolha de informação incluiu recolha documental, entrevistas $(>30)$ e inquérito por questionário $(n=70)$. Ainda que corresponda a um estudo abrangente, e face à diversidade e quantidade de agentes que integram a teia da cooperação portuguesa, não deixa de ser, e citando os autores " (...) uma das visões possíveis e um contributo parcial, que não se substitui à reflexão necessária e urgente por parte dos intervenientes no setor sobre os caminhos a seguir pela cooperação portuguesa e às subsequentes decisões nesse âmbito." (p. 10). O carácter parcial emerge, por exemplo, pelo facto de não incluir de forma alargada o trabalho das UPP e dos respetivos atores. A título de exemplo, no total de entrevistas realizadas, apenas duas estão associadas à academia. Neste sentido, o presente estudo surge como complemento a este esforço integrado de "Promover a análise e investigação independentes em Portugal sobre estas matérias, dando contributos para uma reflexão estratégica no setor da cooperação para o desenvolvimento" (p. 10).

${ }^{3}$ A definição da população institucional em estudo partiu da consulta das Universidades identificadas no sitio oficial da Direção Geral do Ensino Superior: 15 Universidades, 77 Unidades Orgânicas (cf. http://www.dges.mctes.pt/DGES/pt/Estudantes/Rede/Ensino+Superior/Estabelecimentos/ Rede+Publica).

${ }^{4} \mathrm{~A}$ destacar o critério de "delimitação temporal" - protocolos que presentemente estejam a decorrer e/ou que se iniciaram depois de 2000. Esta janela temporal foi definida com base nos objetivos de desenvolvimento do milénio (Faria, 2014). Salienta-se, ainda, que foi também a partir de 2003 que houve uma maior estruturação e profissionalização da cooperação portuguesa, visível através da criação do Instituto Português de Apoio ao Desenvolvimento (2003) e da publicação do documento "Uma Visão Estratégica para a Cooperação Portuguesa" em 2006 pelo Instituto Português de Apoio ao Desenvolvimento e o Ministério dos Negócios Estrangeiros (https://www.instituto-camoes.pt/images/cooperacao/visao_estrategica_para_cp.pdf).

${ }^{5} \mathrm{~A}$ opção metodológica de não identificar as UPP em causa sustenta-se na limitação assumida da constituição da base de dados, que se constitui como uma base de dados parcial, apesar do esforço rigoroso e sistemático levado a cabo aquando a sua constituição. Para além disso, o percurso investigativo permitiu reconhecer o cunho fortemente competitivo interinstitucional existente neste domínio. No sentido de evitar que este estudo seja lido como "propaganda" ao trabalho de internacionalização de IES específicas, optou-se por não identificar as instituições em causa, focando a argumentação e o pensamento na natureza das dinâmicas vividas e refletidas no âmbito dos protocolos estabelecidos. 
${ }^{6}$ Concentração de instituições e de outras entidades públicas e privadas com atividades complementares, o que facilita e potencia a colaboração, e desta forma, o sucesso do trabalho realizado (cf. http://www.instituto-camoes.pt/activity/o-que-fazemos/cooperacao/atuacao/programamos/ clusterses).

${ }^{7}$ Dos 189 protocolos, treze protocolos não foram categorizados de acordo com o domínio do saber envolvido, devido ao caracter genérico do protocolo em causa, e à impossibilidade de aceder a informação mais detalhada sobre os mesmos.

${ }^{8}$ Considerando as regras de financiamento associadas ao Camões I.P., a não identificação da intervenção desta agência foi considerada, em termos de codificação, como ausência de apoio/ participação.

${ }^{9} \mathrm{~A}$ base de dados obtida constitui-se forçosamente como um instrumento de acesso parcial a um cenário global, sobretudo devida a dificuldades de acesso à informação por parte da investigadora. Estes constrangimentos metodológicos encontram-se problematizados de forma mais específica em Silva-Lopes et al. (2016), mas estarão associados sobretudo à falta de acervos institucionais completos e atualizados, lacuna identificada igualmente por Ferreira, Faria e Cardoso (2015) no domínio da cooperação para o desenvolvimento, e, também, à pressão e competição interinstitucional gerada pelo presente paradigma da Economia do Conhecimento (Guilherme \& Dietz, 2015), que estarão na base da frequente morosidade associada à obtenção das autorizações para consulta de informação específica associada aos protocolos de cooperação identificados.

${ }^{10}$ Neste estudo, por limitações de espaço, evidenciam-se apenas as ameaças e fragilidades que foram referidos pelos stakeholders de ambos os protocolos que constituem o cluster intrainstitucional. Salienta-se, no entanto, que os participantes referiram igualmente aspectos positivos externos (oportunidades) e internos (mais-valias) às atividades protocoladas. Optou-se neste artigo por explorar os factores associados ao eixo "negativo" na medida em que importa sobretudo investir no estudo aprofundado destes para identificar e/ou desenvolver recomendações que permitam a superação e/ou minimização dos mesmos. O facto de $88 \%$ do discurso transcrito e categorizado corresponder a ameaças e fragilidades, em detrimento dos $12.35 \%$ associados às oportunidades e mais-valias, evidencia bem o contexto de trabalho de desafio acrescentado que o trabalho, no âmbito de protocolos de CID, representa.

${ }^{11}$ A nível investigativo sugere-se a replicação do estudo para outros agentes da academia (nomeadamente instituições públicas de ensino superior politécnico, e instituições de ensino superior privadas, no sentido de ampliar os resultados da investigação reportada.

\section{Agradecimentos}

O trabalho associado ao presente artigo foi financiado por Fundos Nacionais através da FCT Fundação para a Ciência e a Tecnologia, I.P., no âmbito do projeto "Educação e Formação em Ciências em contexto de cooperação internacional para o desenvolvimento (CID): políticas e práticas sustentáveis potenciadas pela investigação" (SFRH/BPD/100330/2014) e por Fundos Nacionais através da FCT - Fundação para a Ciência e a Tecnologia , I.P., no âmbito da celebração do contrato-programa previsto nos números 4,5 e 6 do art. 23. ${ }^{\circ}$ do D.L. n. ${ }^{\circ}$ 57/2016, de 29 de agosto, alterado pela Lei n. ${ }^{\circ}$ 57/2017, de 19 de julho.".

Uma palavra de agradecimento a todos os informantes que participaram neste estudo e, em particular aos revisores e amigos críticos do artigo, na estruturação do pensamento sobre o enfoque do mesmo.

\section{Referências}

Allouche, J., \& Schmidt, G. (1999). Os instrumentos fundamentais da decisão estratégica. Lisboa: Vislis Editores.

Alonso, A., \& Glenni, J. (2015). What is development cooperation? Development cooperation forum policy briefs. New York: United Nations. 
Alves, J., Marques, M. J., \& Saur, I. (2004). O papel das redes de cooperação na promoção da inovação e na modernização de clusters: o caso do projecto "Casa do Futuro." Estudos Regionais, 6(6), 2743.

Brandenburg, U., \& de Wit, H. (2015). The end of internationalization. International Higher Education, $62,15-17$

Creswell, J. W. (2014). Research design: quantitative, and mixed methods approaches (4th ed.). Thousand Oaks, CA: Sage.

Charmaz, K. (2014). Co-constructing grounded theory (2nd edition). Thousand Oaks, CA: Sage.

Darling-Hammond, L. (2017). Teacher education around the world: What can we learn from international practice? European Journal of Teacher Education, 40(3), 291-309.

Faria, R. (2014). A Cooperação Portuguesa no contexto da Cooperação Internacional para o Desenvolvimento (1998-2012): um ensaio de modelização. (Tese de doutoramento não publicada). Coimbra: Universidade de Coimbra, Faculdade de Letras.

Ferreira, P. M., Faria, F., \& Cardoso, F. J. (2015). O papel de Portugal na arquitectura global do desenvolvimento: opções para o futuro da Cooperação Portuguesa. Lisboa: IMVF \& ECDPM.

Goldstein, H. (2003). Education for all: the globalization of learning targets. Research Intelligence, 82, 18-22.

Gomes, C.A. (2018). Eu e o outro a UNESCO e a educação comparada. Revista Lusófona de Educação, $41,125-138$.

Guilherme, M., \& Dietz, G. (2015). Da universidade à pluriversidade: reflexões sobre o presente e o futuro do ensino superior. Revista Lusófona de Educação, 31, 201-212.

Hamza, A. (2010). International experiences: an opportunity for professional development in Higher Education. Journal of Studies in Higher Education, 14(1), 50-69.

Hodson, D. (2003). Time for action: science education for an alternative future. International Journal of Science Education, 36(6), 645-670.

IPAD. (2010). Desenvolvimento de capacidades: linhas de orientação para a cooperação portuguesa. Lisboa: IPAD.

OCDE (2006). The challenge of capacity development: working towards good practices. Paris: OCED. Disponível em http://www.fao.org/fileadmin/templates/capacitybuilding/pdf/DAC_paper_final.pdf.

OCDE (2014). Building a Teaching Profession: lessons from around the world. Paris: OCDE.

OCED. (2015). OECD Development Co-operation Peer Reviews: Portugal 2016. Paris: OCDE.

Lopes, B., \& Diogo, S. (2019). Institutional Challenges in monitoring and evaluating cooperation protocols between higher education institutions in Portuguese speaking countries. Livro de atas INTEND 2019 (pp. 11563-1566). Valência, Espanha: INTEND.

Martins, I. P., Pedrosa, M. A., Ferreira, A. J., \& Simões, M. O. (2014). Chemistry and Education for sustainability: foundations and curricular proposals for East Timor. Educación Química, 17, 20-29.

Sangreman, C. (2015). O cluster como instrumento teórico e prático de inovação na cooperação para o desenvolvimento. Lisboa: CeSA. 
Silva-Lopes, B., Albergaria-Almeida, P., Costa, N., \& Callapez, P. (2016). Cooperação para o desenvolvimento no âmbito da Educação em Ciências - estudo das atividades protocoladas entre Portugal e países africanos de língua oficial portuguesa. Livro de atas do Fórum Internacional África, Cooperação, Educação e Desenvolvimento, FIACED (pp. 81-89). Penafiel, Portugal: Instituto Superior de Ciências Educativas do Douro.

Silva-Lopes, B., Lucas, M., Albergaria-Almeida, P., \& Martinho, M. (2017). Training Timorese Science Teachers in the context of international cooperation: what role could ICT play? Conexão Ciência, 12(2), 416-423.

Stein, S., Andreotti, V. O., \& Susa, R. (2016). 'Beyond 2015', within the modern/colonial global imaginary? Global development and higher education. Critical Studies in Education, 1-21.

UNESCO (2016). Education 2030: Incheon Declaration and Framework for Action towards inclusive and equitable quality education and lifelong learning for all. Paris: UNESCO.

Betina Silva-Lopes

Universidade de Aveiro, CIDTFF (Portugal)

Email: blopes@ua.pt

Orcid: https://orcid.org/0000-0003-0669-1650

Correspondência

Universidade de Aveiro, Departamento de Educação e Psicologia

Campus Universitário de Santiago, 3810-193 Aveiro, Portugal

Data de submissão: Março 2019

Data de avaliação: Maio 2019

Data de publicação: Abril 2020 